
allemande

47-1| 2015

L'Allemagne et l'Europe | La déprise de l'Empire napoléonien en Allemagne en 1813

\title{
La réception mémorielle de la campagne de Russie des soldats westphaliens après 1813
}

Anika Bethan

\section{OpenEdition}

\section{Journals}

Édition électronique

URL : https://journals.openedition.org/allemagne/474

DOI : 10.4000 /allemagne.474

ISSN : 2605-7913

Éditeur

Société d'études allemandes

Édition imprimée

Date de publication : 26 juin 2015

Pagination : 177-184

ISSN : 0035-0974

Référence électronique

Anika Bethan, «La réception mémorielle de la campagne de Russie des soldats westphaliens après 1813 », Revue d'Allemagne et des pays de langue allemande [En ligne], 47-1 | 2015, mis en ligne le 13 décembre 2017, consulté le 23 mai 2021. URL : http://journals.openedition.org/allemagne/474 ; DOI : https://doi.org/10.4000/allemagne.474 


\title{
La réception mémorielle de la campagne de Russie des soldats westphaliens après 1813
}

\author{
- Anika Bethan*
}

Malgré sa très courte existence, le royaume de Westphalie est un intéressant cas d'étude pour les questions relatives aux phénomènes de sortie de guerre et de mémoire des conflits. Cette contribution se concentre sur la destinée des soldats westphaliens jusqu'en 1813 et cherche à montrer comment ils devinrent les sujets et les objets d'une mémoire collective. Au-delà, elle envisagera la manière dont on commémora en Westphalie la fin de la période napoléonienne, et dans quelle mesure le souvenir en fut affecté par la nouvelle dimension que celle-ci avait donnée à la guerre. Comment la perception des guerres napoléoniennes et les développements dynastiques intervenus dans les États allemands s'y reflètent-ils? Il est important de donner de ces sujets une analyse multipliant les perspectives différentes, dans la mesure où l'appréciation de la période fut souvent dominée par le point de vue national ou nationaliste, où se surimposaient perspectives locales et approches régionales et qui manquait d'un regard surplombant sur ces événements, bref d'une interprétation d'ensemble.

\section{L'armée westphalienne}

En 1807, Jérôme Bonaparte, le plus jeune des frères de Napoléon, prit la couronne de Westphalie. Il avait obligation de fournir 25000 hommes à la Grande Armée, d'où le fait qu'il instaura la conscription, telle qu'elle fonctionnait en France, avec ses inconvénients mais aussi ses avantages, tels que les nouvelles opportunités de carrière par la voie militaire et les secours versés aux soldats blessés ou invalides. De plus, Jérôme Bonaparte renforça le lien traditionnellement bien ancré entre le souverain et l'armée, qui avait des vertus stabilisatrices sur le plan social. Il distribua des médailles, des décorations militaires, et ce faisant il fixa un cadre proprement westphalien d'intellection et d'appréhension de ces conflits ${ }^{(1)}$. Les honneurs ainsi dispensés n'étaient

* Docteur en histoire, lectrice du DAAD à l'Université Ain Shams, Le Caire.

1 M.-G. Biennais, Recueil des ordres français et étrangers. Dessins originaux. Geschenk an den Kaiser, s.l.n.d. (Bibliothèque Marmottan Paris). 
pas seulement des pensions et des récompenses. Ils encourageaient une perception positive du nouvel outil militaire mis en place par le roi Jérôme et entretenaient les liens des habitants avec leur Vaterland westphalien. L'ancienne armée composée de mercenaires devint effectivement une armée nationale toute nouvelle ${ }^{(2)}$. Les régiments de Hesse, du Brunswick, du Hanovre et de Prusse marchaient désormais sous l'uniforme westphalien parmi les autres troupes de Napoléon, et ils firent les campagnes d'Espagne (1808-1811) et de Russie (1812) avant de défendre le Nord de l'Allemagne, en 1813 , et de combattre à Leipzig sous le drapeau français. Après cette bataille, le roi Jérôme s'étant enfui, l’armée westphalienne se désintégra.

Les anciennes familles régnantes que Napoléon avait expulsées en 1806 retournèrent à leurs capitales, bien que leurs armes y fussent pour peu de chose. C'était principalement le fait des troupes prussiennes et autrichiennes qui s'étaient battues pour chasser les Français des régions du centre de l'Allemagne. Leur prétention à reprendre leur souveraineté n'avait donc rien d'évident en elle-même. Ainsi, l'électeur de Hesse fut tellement fustigé par la Prusse qu'il dut justifier de ses prétentions à revenir au pouvoir. Il y parvint en se présentant comme le meilleur garant de l'ordre public. Avant même la fin brutale du gouvernement du royaume de Westphalie, les désertions massives et les passages de troupes, quantitativement très importants, n'avaient pas manqué de susciter des troubles populaires. Les impôts ne rentraient plus et les ordres de l'administration suscitaient des conduites de refus, au point de provoquer l'escalade et de changer nombre de contentieux en véritables émeutes. L'électeur sut apaiser la situation en annulant purement et simplement toutes les provisions et règlements édictés par l'ancien royaume de Westphalie et en sollicitant la loyauté des populations hessoises. Une fois restaurée, l'ancienne dynastie de Hesse réussit à rétablir une administration efficace et à recruter une nouvelle armée destinée à poursuivre les guerres de libération contre Napoléon, car une participation active à ces opérations militaires aux côtés des Alliés était essentielle à l'avenir de l'électorat de Hesse.

De ce point de vue, il n'est pas étonnant que le transfert de l'armée westphalienne au sein des nouvelles troupes recréées, à l'échelle des petits États, par les anciens souverains, se soit déroulé sans opposition ${ }^{(3)}$. Néanmoins, si les anciens officiers et soldats n'avaient pour la plupart aucune suite judiciaire à redouter, la question de leur promotion restait problématique. Les gouvernements successeurs du royaume de Westphalie ne reconnurent aucun titre ou rang établi sous le règne de Jérôme, et il en fut de même pour les pensions et les récompenses ${ }^{(4)}$.

\section{Le mythe des guerres de libération et l'héritage de la Westphalie}

La mère patrie pour laquelle les soldats westphaliens combattaient durant les guerres de libération, de 1813 à 1815, n'était plus la même que celle qu'ils avaient servie derrière

2 Bettina Severin-Barboutie, « Vom freiwilligen Söldner zum wehrpflichtigen Untertan », in: Michael Eissenhauer (dir.), König Lustik!? Jérôme Bonaparte und der Modellstaat Königreich Westphalen, Munich, Hirmer, 2008, p. 120-126. Plus récemment, en français, voir la biographie de Jacques-Olivier Boudon, Le roi Jérôme. Frère prodigue de Napoléon (1784-1860), Paris, Fayard, 2008.

3 Landesarchiv Münster (LAMü) : Regierungskommission Münster MS B 102, Nr. 106, Besitznahme der Kgl. Preuß. Westphälischen Provinzen 1813.

4 Carl Wilhelm Wippermann, Kurhessen seit dem Freiheitskriege, Cassel, p. 8. 
l'empereur des Français en amont de 1813. Un énorme appareil de propagande mis en route par les Alliés, particulièrement par la Prusse, chercha à atteindre la population civile et à lui "vendre " l'idée nationale allemande, en présentant la France comme l'archétype de l'ennemi ${ }^{(5)}$. Les souverains l'affirmèrent pareillement, en cherchant à mêler cela avec l'ancien patriotisme dynastique de leurs sujets. Ainsi, alors que les anciennes troupes westphaliennes se battaient pour l'idée d'une nation allemande, leur affiliation régionale n'en était pas moins importante. Mais aussi bien la continuité dynastique que la référence à l'Allemagne rendaient impossible de considérer positivement leur tout récent passé militaire au service de la France.

Mais d'autre part, les incitations " régionales » ou " nationales » évitaient l'une et l'autre de placer explicitement les soldats westphaliens du côté d'une France « ennemie héréditaire »(Erbfeind). Pendant les cérémonies et rituels commémoratifs du $\mathrm{XIX}^{\mathrm{e}}$ siècle, comme l'anniversaire de la bataille de Leipzig, il est manifeste que la période westphalienne fut passée sous silence ${ }^{(6)}$, de sorte qu'il est possible de parler d'une sorte de damnatio memoriae du côté des principautés allemandes issues de la décomposition du royaume. Dans ces cérémoniaux, qui cherchaient à faire sentir la force des liens entre le souverain et son peuple, les vétérans et anciens soldats reçurent un rôle important ${ }^{(7)}$, mais sans que leur passé au service de la Westphalie fût jamais mentionné( ${ }^{(8)}$. Leurs vertus militaires ne devaient être associées qu'aux seules guerres de libération. Mais parallèlement, il faut penser que l'armée était étroitement liée à la population civile (le recrutement nourrissait directement un grand nombre de familles) et au rayonnement du souverain. De ce fait, il n'était pas possible non plus d'en donner un tableau négatif. Les sources suggèrent que cette difficulté resta récurrente pendant au moins cinq décennies. Ainsi, encore en 1863, un article de la Helmstedter Kreisblatt affirma qu'il n'y avait pas de "mémoire adéquate » (keine angenehmen Erinnerungen $»)$ des troupes westphaliennes ${ }^{(9)}$.

\section{Mémoire régionale et mémoire locale}

S’affronter au passé militaire du royaume de Westphalie était essentiel pour les vétérans, non seulement pour ce qui relevait de leur place dans la société, du prestige qui leur était reconnu, des honneurs qui leur étaient rendus, mais aussi pour des questions économiques. De nombreuses réclamations furent déposées relativement aux paies qui n'avaient pas été versées pendant la période westphalienne ou bien aux pensions. Les gouvernements successeurs n'avaient pas l'intention de satisfaire ces demandes, même si la convention fédérale de Francfort avait déclaré que les souverains rentrés dans leurs États étaient les héritiers des lois du royaume de Westphalie au 2 décembre

5 Karen Hagemann, Männlicher Muth und Teutsche Ehre. Nation, Militär und Geschlecht zur Zeit der Antinapoleonischen Kriege Preußens, Paderborn, Ferdinand Schöningh, 2002, p. 29-35; Jörn LEONHARD, Bellizismus und Nation. Kriegsdeutung und Nationsbestimmung in Europa und den Vereinigten Staaten 1750-1914, Munich, Oldenbourg, 2008, p. 232 et p. 239.

6 «Die fünfzigjährige Gedenkfeier der Schlacht bei Leipzig in verschiedenen Städten Kurhessens », Kasseler Zeitung, 25 octobre 1863.

7 K. Hagemann, Mannlicher Muth und Teutsche Ehre (note 5), p. 475-481.

8 "Décret de l'Électeur », Kasselsche Allgemeine Zeitung, 19 octobre 1816.

9 Helmstedter Kreisblatt, 4 février 1863. 
1813, et avait ordonné la création d'une commission commune pour traiter des questions judiciaires que l'alternance des pouvoirs poserait. Celle-ci ne devait cependant pas se réunir régulièrement avant 1832. L'initiative vint de Berlin, la Prusse ayant été le seul État à reconnaître le royaume de Westphalie dans le cadre du traité de Tilsit en 1807, et ne pouvant donc l'absoudre de sa responsabilité juridique dans les matières concernant les territoires de l'Ouest qui lui étaient revenus après 1813. Le Hanovre était également touché par cette situation et il avait dû collaborer avec la Prusse pour ce qui concernait la répartition des territoires décidée au congrès de Vienne. Suivant l'exemple prussien, le Hanovre régla le sort juridique de ses sujets anciens soldats de la Westphalie dans son Bulletin ministériel (Ministerialbekanntmachung) du 15 octobre 1835, et jugea ces réclamations légitimes. Deux ans plus tard, le duché de Brunswick adopta les mêmes régulations, se réclamant explicitement de l'exemple de la Prusse et du Hanovre ${ }^{(10)}$. Cependant, il faut remarquer que la diminution substantielle du nombre de requérants encore en vie avait facilité le choix des autorités. En Brunswick, les demandes avaient fondu jusqu'à seulement 3 ou $4^{(11)}$. Seule la Hesse-Cassel refusa avec véhémence de satisfaire ces réclamations, ce qu'elle justifia en rappelant que Napoléon avait violé le droit des gens en occupant le territoire hessois, ce qui annulait automatiquement l'engagement de chaque Westphalien ${ }^{(12)}$.

À la suite de l'échec des premières demandes, qui furent présentées immédiatement après la chute du royaume de Westphalie en 1813, une nouvelle vague de réclamations émergea dans les années 1830 . On en a la preuve dans les archives judiciaires de Göttingen, où on décompte 104 requêtes déposées pendant cette période ${ }^{(13)}$. 65 requérants étaient d'anciens soldats westphaliens, les autres demandes émanant d'héritiers. Les demandes concernaient surtout les années 1806 et 1807, et particulièrement aussi 1813, durant laquelle les soldes n’avaient pas été versées. Ces demandes sont révélatrices de deux phénomènes concernant la mémoire du royaume de Westphalie. D’abord que l'attitude dilatoire et hésitante du gouvernement eut pour effet de conserver ce passé vivant pour nombre d'anciens militaires et de familles de vétérans. À terme, les autorités furent obligées de s'affronter sans détour à cette période de l'histoire proche, pas avant les années 1830 sans doute, et dans certains cas comme l'électorat de Hesse, pas avant $1865^{(14)}$. D'autre part, les sources montrent que les plaignants et pétitionnaires faisaient surtout usage de termes neutres. Il n'y a que très peu de requêtes (11 sur 106 à Göttingen, par exemple) où ils aient cherché à justifier leur engagement dans l'armée, ou simplement tenté de décrire en détail leur situation. Cela suggère que pour les auteurs de ces demandes, il était admis que cette période était bien connue de leurs interlocuteurs, et que leur participation à des grandes batailles valait comme

10 Staatsarchiv Wolfenbüttel (StAW) : IV Hs 18 Militärwesen, nº 500, requête du 27 janvier 1837.

11 StAW, HS $18, \mathrm{n}^{\circ} 497$, requête du 20 mars et 23 novembre 1833.

12 Eike Erdel, «Der westphälische Domänenstreit », in: Helmut Burmeister (dir.), König Jérôme und der Reformstaat Westphalen. Ein junger Monarch und seine Zeit im Spannungsfeld von Begeisterung und Ablehnung, Hofgeismar, Verein für Hessiche Geschichte und Landeskunde, 2006, p. 379-389.

13 Stadtarchiv Göttingen (StdAGö) : AA Militärsachen, n 39, 1835-1839.

14 Hauptstaatsarchiv Hannover (HstAM): dossier du secrétaire de police de Cassel, Gieseler, 23 juin 1865 (Aktenbestand 12 Kriegsministerium 12 a 39 Gagereklamationen ehemaliger Offiziere und Soldaten des Königreich Westphalen 1831-65). 
une preuve de mérite personnel, sans la moindre affiliation qui eût une portée politique. Aussi la plupart contiennent-elles une liste des combats auquel le requérant a pris part ${ }^{(15)}$, voire la liste de ses décorations westphaliennes ${ }^{(16)}$. Pratiquement toujours, ils se désignent d'ailleurs comme des soldats « westphaliens".

Si le gouvernement du Hanovre a tenté de trouver un terrain d'accommodement avec ses sujets anciens combattants de la Westphalie, via son Bulletin ministériel de 1835 , cela ne signifie pas bien sûr qu'il donna satisfaction à toutes les demandes. Dans les sources conservées à Göttingen, seules $40 \%$ des demandes ont une suite favorable. Les justifications présentées par les requérants n'étaient pas, de ce fait, si importantes. À la base, le Hanovre refusait de reconnaître les décorations westphaliennes, les pensions accordées par l'armée westphalienne et toutes les demandes émanant de familles de soldats qui avaient disparu dans la guerre d'Espagne ou la campagne de Russie. Ces dernières avaient eu pourtant un grand impact sur la société allemande, car «il n'y avait presque pas une seule famille dans le pays qui ne portât pas le deuil d'un de ses membres qui avait eu à souffrir une fin atroce dans les étendues glacées de Russie » ${ }^{(17)}$. Le refus de verser la moindre compensation financière n'était pas seulement un problème pour les familles, mais cela posait aussi des questions juridiques spécifiques. En particulier concernant la campagne de Russie, pour laquelle les renseignements fiables manquaient concernant le sort des soldats portés manquants. Beaucoup étaient morts non pas dans des hôpitaux militaires, mais dans la retraite désordonnée de l'hiver 1812-1813, sans laisser la moindre trace. Pour les héritages, c'était là une grosse difficulté et les autorités ne tardèrent pas à se rendre compte qu'il fallait trouver à ces cas une solution rapide.

Aussi, dès 1815, le Hanovre envoya un représentant en Russie, en charge d'étudier le destin des soldats portés disparus ${ }^{(18)}$. Deux ans plus tard, celui-ci remit des listes de noms qui servirent de base à l'établissement de certificats de décès, et qui permirent aussi bien de mettre en place des mesures adéquates relativement aux questions patrimoniales non réglées que de mettre en route des hommages à ces morts. Néanmoins, ces listes ne permettaient d'identifier qu'un nombre restreint de soldats tombés en Russie, de sorte que le sujet demeura présent dans le débat public des États successeurs de la Westphalie plusieurs décennies durant ${ }^{(19)}$. Au niveau gouvernemental, dans ces procédures, on évitait toute référence explicite à la Westphalie. Dans les notices, bulletins et autres documents conservés, seul le terme « en Russie " peut être trouvé, terme qui permettait de ne pas préjuger de l'engagement des militaires disparus dans un camp ou un autre.

15 StdAGö, Militärsachen, requête de M. Ziegler, 6 décembre 1835, ou H. Wepner, 8 décembre 1835, ou encore H. Schönhardt, 22 novembre 1835.

16 Ibid., requête de J.H. Ruder, 12 novembre 1835.

17 « da sich fast keine Familie im Lande befand, welche nicht Mitglieder beweinte, die in Rußlands Eisgefilden einen schmählichen Tod gefunden [...]», in : Friedrich August Karl von Specht, Das Königreich Westphalen und seine Armee im Jahr 1813, sowie die Auflösung derselben durch den kaiserlich russischen General Graf A Czernicheff, Cassel, 1848, p. 43.

18 StdAGö, AA-D, n 6103, et AA Kriegssachen Napoleonische Kriege nº 12.

19 Voir le cas de Johann Klapps, d’Ippinghausen, dans la Kasselsche Allgemeine Zeitung du 20 juin 1838. 


\section{Romans nationaux}

La propagande suscitée par les guerres de libération, en particulier l'interprétation des événements de type national-allemand telle qu'elle se développa dès le début du $\mathrm{XIX}^{\mathrm{e}}$ siècle, prenant son origine tout spécialement en Prusse, ne laissait guère l'espace disponible aux soldats westphaliens. Cela concorde avec l'approche de l'historiographie allemande, pour laquelle la catastrophe de Russie était une sorte de juste châtiment pour Napoléon. L'historiographie locale et régionale des État successeurs de la Westphalie commença à mettre en cause ces prodromes ${ }^{(20)}$, en séparant notamment les souffrances endurées par les populations des règlements politiques, et en distinguant les mérites militaires des troupes westphaliennes de celles de leur " roi-souillon », Jérôme Bonaparte ${ }^{(21)}$.

Les anciens soldats westphaliens ne pouvaient pas se positionner dans le cours de l'histoire allemande en construction, ils se situèrent donc plutôt dans une perspective régionale. Il y avait aussi les développements d'une historiographie moderne, un sujet important et populaire hérité de la période napoléonienne en Allemagne - mais aussi ailleurs. Or, pour les historiens anglophones ou francophones, à rebours de la lecture prussienne dominante des événements allemands, le problème n'existait pas. Bien qu'ils n'eussent aucun intérêt particulier pour le cas de la Westphalie, ils mentionnaient Jérôme et ses troupes, en référence à la retraite de Russie, comme des membres à part entière de la Grande Armée, comme "nos braves " pour reprendre le terme de Labaume ${ }^{(22)}$, des soldats vertueux, quoique pas toujours parfaitement loyaux vis-à-vis de leur roi ${ }^{(23)}$.

Avant 1820 , les historiens britanniques ne possédaient pas une connaissance très solide de l'histoire récente du continent européen, en dehors de ce qui concernait les affaires d'Espagne et la Peninsular War. Néanmoins, dans leurs descriptions de la campagne de Russie, ils présentèrent les unités westphaliennes, en soulignant les pertes importantes qu'elles avaient subies et les qualités militaires dont elles avaient fait preuve ${ }^{(24)}$. Ces récits eurent une certaine prise sur les régions de l'ancienne Westphalie, par l'intermédiaire de traductions qui furent lues dans les veillées. Une signification particulière fut aussi accordée aux récompenses militaires délivrées sous Napoléon III. En 1857, l'empereur fit frapper une « médaille de Sainte-Hélène » en

20 Barbara Besslich, Der deutsche Napoleon-Mythos. Literatur und Erinnerung 1800 bis 1945, Darmstadt, WBG, 2007, p. 270.

21 Parmi bien d'autres auteurs, citons ainsi: Karl Venturini, Rußlands und Deutschlands Befreiungskrieg von der Franzosenherrschaft unter Napoleon Buonaparte in den Jahren 1812 und 1815, Leipzig/ Altenburg, 1816; Friedrich SAALFELD, Rußlands und Deutschlands Befreiungskrieg von der Franzosenherrschaft unter Napoleon Buonaparte in den Jahren 1812 und 1815, Leipzig/Altenburg, 1815; August Wilhelm ReHBerg, Zur Geschichte des Königreichs Hannover in den ersten Jahren nach der Befreiung, Göttingen, 1826; Albert HüNE, Geschichte des Königreichs Hannover und Herzogthums Braunschweig, Hanovre, 1830.

22 Eugène Laваume, La campagne de Russie. Le récit d'un officier de la Grande Armée, Paris, 1813-1814, p. 55 et p. 138.

23 Adolphe Thiers, Histoire du Consulat et de l'Empire, Paris, 1843-1862, t. 6, p. 234.

24 Walter ScotT, Life of Napoleon Buonaparte with a preliminary view of the French Revolution, Édimbourg, 1827, p. 609. 
hommage aux anciens compagnons d'armes de son oncle ${ }^{(25)}$. Ce fut la première reconnaissance officielle du sacrifice des troupes westphaliennes qui émergea après un peu plus de quarante ans, mais de la part du gouvernement français, les parties allemandes refusant bien sûr cette distinction. Leur mémoire, longtemps confinée historiquement au niveau local et régional, s'était enfin ouverte à un cadre référentiel plus vaste.

\section{Conclusion}

Ainsi que nous l'avons vu, les soldats westphaliens étaient un enjeu d'importance pour les anciens souverains devenus les nouveaux maîtres dans l'Allemagne de 1813. Ces derniers devaient légitimer politiquement leurs revendications territoriales en prenant part aux guerres de libération et en rétablissant l'ordre intérieur dans leurs États. Mais si les hommes de troupe et les officiers de l'ancienne Westphalie n'avaient à craindre aucune suite judiciaire, leurs états de service ne pouvaient faire l'objet d'une reconnaissance officielle. Bien qu'ils ne fussent jamais mentionnés publiquement, ces anciens combattants avaient un passé qui resta vivant dans la mémoire de la population locale. C'est une évidence si l'on se fie aux longues procédures judiciaires, à la mémoire empêchée et aux échos des journaux et des bulletins de l'époque. Dans ce cadre, le souvenir de la campagne de Russie jouait un rôle très significatif, car l'impact du désastre militaire était encore palpable à tous les degrés de la société allemande. À terme, ce sont les historiographies étrangères, et la médaille d'honneur émise en France sous le Second Empire, qui devaient fournir un nouveau cadre référentiel permettant de situer dans l'Histoire l'expérience des troupes westphaliennes.

Traduit de l'anglais par Nicolas Bourguinat

\section{Résumé}

L'article se centre sur l'expérience des soldats issus de "l'État modèle » de Napoléon en Allemagne, le royaume westphalien, et spécialement celle qu'il firent de la campagne de Russie. Elle devait avoir pour eux un rôle décisif après les guerres de 1813, mais les politiques de la mémoire mises en place par les États allemands successeurs empêchèrent que les vétérans puissent prétendre à une commémoration des événements. L'idée d'une partition amis/ennemis qui s'était formée pendant les guerres de libération interdisait que la période napoléonienne en Westphalie soit située précisément dans l'interprétation officielle des événements. Aussi le processus de mémoire collective des anciens soldats n'intervenait-il qu'à des niveaux sociaux ou spatiaux bien spécifiques. Il semble avoir été un outil de cohésion, ce qui conduit à identifier les contextes et périodes historiques au sein desquels le vocable «westphalien » a pu s'imposer pour désigner ces hommes, par opposition à ceux où il aurait été volontairement évité ou se serait vu substituer d'autres dénominations.

25 Voir Sudhir Hazareesingh, La Saint-Napoléon. Quand le 14 juillet se fêtait le 15 août, Paris, Tallandier, 2007, p. 99-124. 


\begin{abstract}
The article focusses on the militaries of Napoleon's 'model state', Kingdom of Westphalia (1807-1813), for whom the experiences of the Russian Campaign played a decisive role after 1813. However, the policy of remembrance implemented by the governments of the German territorial states obstructed a commemoration of this event for the veterans, because the idea of a friend-enemy-constellation, which was formed within the wars of liberation against Napoleon, hindered a clear location of the Westphalian period in the official interpretation of events. Thus, collective memory processes of the militaries occurred only at different social and spacial levels and show themselves as processes of cohesions, which led to conclusions about when and under which context these cohesions under the self- or foreign-designation of 'Westphalian' appeared and when such designations were explicitly avoided or replaced by others.
\end{abstract}

